\title{
A deterministic method to integrate triangular meshes of different resolution
}

\author{
Giorgio Agugiaro ${ }^{\mathrm{a}, *}$, Thomas H. Kolbe ${ }^{\mathrm{b}}$ \\ a Department of Architecture, Urban Planning and Surveying, Università degli Studi di Padova, Italy \\ ${ }^{\mathrm{b}}$ Department for Geodesy and Geoinformation Science, Technische Universität Berlin, Germany
}

\section{A R T I C L E I N F O}

\section{Article history:}

Received 30 July 2010

Received in revised form 10 May 2012

Accepted 15 May 2012

Available online 20 June 2012

\section{Keywords:}

DSM

DTM

Multi-resolution data integration

Surface generation

Laser scanning

\begin{abstract}
A B S T R A C T
As a result of new sensors and methodologies that allow for faster and more accurate data acquisition, the production of highly detailed 3D models is becoming part of the standard workflow in many disciplines architecture, cultural heritage, urban planning, and others. At the same time, new related problems have emerged concerning the management of the resulting large datasets and the integration of data coming from different sources. Data integration among heterogeneous datasets can lead to a variety of errors, and the reasons for these errors are multiple including geometric, topological or semantic incompatibilities, different resolutions and accuracies, and data acquired at different times. To overcome such errors, the integration of heterogeneous datasets requires appropriate homogenisation techniques.

This article presents a new deterministic approach for the integration of a high resolution surface model and a lower resolution surface model, both given as triangular meshes embedded in 3D space, by means of a transition surface. The method can be applied after both datasets have been aligned. The derivation of the transition surface exploits extra data that are typically available in the high resolution model. These extra data (e.g. quite common when using a laser scanner) must be $2.5 \mathrm{D}$ and located around the high resolution model, they represent a sort of planimetric buffer around the high resolution object that can be thought as a "collar". The proposed method generates a geometrically and topologically sound and continuous $2.5 \mathrm{D}$ surface that integrates the two models using the data contained in the collar. It takes into account the different quality aspects of the low and high resolution models such as point height, point density, and height gradient, and then creates a transition surface that interpolates these aspects between the embedded high resolution model and the surrounding low resolution model. The article also presents some experimental results, obtained from real datasets, and concludes with some remaining issues and possible improvements to the method.

(c) 2012 International Society for Photogrammetry and Remote Sensing, Inc. (ISPRS) Published by Elsevier
\end{abstract} B.V. All rights reserved.

\section{Introduction}

Today it can be assumed that the Earth's surface has been completely mapped. However, the varying methodologies and different sensors and techniques for mapping have led to multiple representations of the Earth's surface - or specific portions of it - with different levels of details. These multiple models often represent the same object in different ways, depending on the origin and the goal for data acquisition. Moreover, the rapid development of new sensors and faster and more accurate acquisition methodologies, along with the increasing number of geo-enabled applications using the growing amount of distributed geo-data, requires multi-resolution, multi-format data integration. Datasets created for a specific task

\footnotetext{
* Corresponding authors. Present address: 3D Optical Metrology Unit, FBK Fondazione Bruno Kessler, Trento, Italy.

E-mail addresses: agugiaro@fbk.eu(G. Agugiaro), thomas.kolbe@tu-berlin.de (T.H. Kolbe).
}

are therefore being merged into larger, integrated, multi-purpose geo-databases.

The reasons for this convergence are multiple, but some general causes can be deduced. First, integration of existing data into a common environment may reduce costs, thus avoiding the need for new data acquisition (and sometimes older datasets might be purposefully used). Second, integration helps reduce inconsistencies that are caused by different object modelling, surveying and production methods. The ability to verify outdated datasets that can be checked against newer ones should not be underestimated.

Examples of the data integration trend are projects like Google Earth or Bing Maps 3D, which have experienced a steady growth in popularity and amount of offered services since their launch few years ago. By integrating data from different sources and resolutions, such applications deliver effective visualisations of large scale models to broad audiences. Another relatively recent example of the importance and usefulness of data integration is the recent boom in 3D city models. The integration of different models like DTMs, buildings, transportation facilities, etc. is an important 
topic that is igniting worldwide large-scale research efforts (Kolbe, 2008). Virtual 3D city models represent, in fact, an important and cost effective tool that is useful in those fields where multidisciplinary approaches are of great importance, such as urban planning and disaster management, although architectural design and cadastre can equally profit from them. A further example is digital terrain modelling, which in recent years has experienced significant improvements, in great part due to new technologies such as digital photogrammetry or Aerial Laser Scanning (ALS) that can quickly output millions of points over the acquired surface and produce a highly detailed terrain model. As a final example, the field of cultural heritage has seen great advances with regard to data acquisition, integration, management and visualisation, e.g. the Rome Reborn project, later implemented also in Google Earth as Ancient Rome 3D layer (Guidi et al., 2007).

While the above mentioned examples have been chosen from heterogeneous frameworks, they all are confronted with similar problems when it comes to data integration. As long as a specific task can be carried out using a single model - or a set of models having the same characteristics - there might be no apparent reasons to consider how one model relates to other different models. However, as soon as two or more heterogeneous datasets must be simultaneously used, problems related to their integration might arise. Reasons for integration inconsistencies typically include: (1) geometric, topological or semantic incompatibilities, (2) "similar" datasets that originate from different sensors and have different resolutions and accuracies, and (3) data that were acquired at different times. Of course, a combination of the above mentioned causes is also possible. A common problem is that high resolution models of sites and objects are created but cannot be integrated with the typically lower resolution models of the surrounding terrain or environment. Given the variety in size and level of detail between the different models, discrepancies can easily emerge during their integration. Typical examples for such integration discrepancies are gaps or cracks at the borders of adjacent datasets - instead of the expected seamless transition - or models at different resolutions which do not fit exactly, like in the case of a house hovering over the underlying terrain model. Despite the issues associated with data integration, the need to integrate different datasets is inevitable, due to both practical and economic circumstances. Hence, the topic is highly relevant, yet no general solution has been proposed.

This article focuses on data integration by means of geometric homogenisation between two heterogeneous datasets. Specifically, it investigates the possibility to perform a local update at selected areas within a wider, however coarser, model by means of a high resolution model. The developed methodology requires that the two input datasets are triangular meshes that have been previously aligned and share, therefore, the same coordinate system. A fundamental requirement of the high resolution model is that it must contain not only data of the represented object (e.g. a building) but also some extra geometrical information around it (e.g. some terrain around the building), as a sort of planimetric buffer, here called "collar". In addition, both the high resolution data of the collar and the low resolution data overlapping the collar must be $2.5 \mathrm{D}$. There are no restrictions for the high resolution object itself (e.g. the building) or for the low resolution data not overlapping the collar.

Please note that in this article much of the reasoning is made keeping in mind that the low resolution model will be considered to be a triangular mesh derived from a digital terrain model (DTM, representing the bare terrain), but actually it might well also be a digital surface model (DSM, representing the terrain and the objects on top of it like houses and trees), or, more generally, it could be any other model, as long as it complies with the initial assumptions and requirements mentioned above and more precisely described in Section 3.1.

Using these criteria, a new deterministic approach is described that allows for integration between a high resolution surface model and a lower resolution surface model by means of a transition surface. The goal is to create a geometrically and topologically sound continuous surface between the two models. This surface connects the models smoothly, without modifications to the actual high resolution object, and permits a transition also in terms of point density. In order to obtain the transition surface, four successive steps are required. First, only the data necessary for the modelling purposes are selected from the input datasets. Second, a new constrained Delaunay triangulation is performed inside the overlapping zone, using the previously selected data in order to guarantee topological continuity between the two models. Third, a spline-based interpolation function is implemented to smooth the transition surface, and fourth, a progressive mesh simplification algorithm is performed on it to selectively reduce data.

In Section 2 some related works are introduced, from which the present approach has taken inspiration. Section 3 represents the main contribution of this article and describes the developed methodology in detail and explains the initial assumptions and the stepwise modus operandi of the methodology. Section 4 contains some notes about the implementation and Section 5 discusses the results of the experiments that were carried out on models from case studies in urban modelling, archaeology and cultural heritage. Section 6 presents the conclusions, some further reasoning and some ideas for future improvements.

\section{Integration of fragmented datasets and related work}

In general, once two datasets have been aligned, inconsistencies resulting from the merge of different geographic datasets can be grouped into two groups (Laurini, 1998): (1) layer fragmentation, in which discrepancies originate in datasets covering the same region but containing different feature classes (e.g. a DTM and a topographic map or a "flying" 3D building on top of it) and (2) zonal fragmentation in which errors may originate from datasets containing similar information but covering spatially disjoint regions (e.g. DTMs belonging to different neighbouring countries). Because of the inexact matching at the boundaries some difficulties occur in order to ensure geometric and topological continuity between the different datasets. Layer and zonal fragmentation are very frequent, considering that different institutions often have different kinds of information on different zones.

Depending on the quantity and quality of available information accompanying and characterising the fragmented datasets (e.g. the presence or absence of semantics), different data integration strategies can be pursued to overcome inconsistencies.

\subsection{Semantic-based integration}

Semantic modelling represents one of the keys to the integration of fragmented geo-objects from different domains (Förstner and Plümer, 1997). The integration process must first identify the various representations of corresponding objects, then their geometric inconsistencies can be corrected and homogenised. As a consequence, the more information that is provided by the semantic layer, the fewer ambiguities that remain for the geometric integration. To this extent, Stadler and Kolbe (2007) discuss the importance of coherently structured semantics and geometry in a model and analyse their correspondence. Essentially, the more that the semantic information and geometries are similarly structured, the higher the level of coherence. 
Koch and Heipke (2006) have proposed an algorithm that uses semantic information in order to integrate DTMs and data coming from a two-dimensional topographic vector datasets, which often include geographical features like roads, rivers, and lakes that contain intrinsic height information to be employed through a proper set of constraints. A lake can be represented, for instance, by a horizontal plane polygon, hence the neighbouring banks must be higher than the water and, in the same way, the slope of a road must not exceed a certain maximum value. Corresponding constraints (e.g. on the height of the lake and of the banks) are derived and enforced according to the semantic information.

\subsection{Geometry-based integration by homogenisation}

In case there is no semantics at all, or semantics and geometry are uncorrelated, the integration cannot rely on connecting (semantic) features to correlate geometries and must be carried out purely on the basis of geometric shape.

Although the definition of a generic and automated homogenisation process is a still subject of research, the integration of fragmented datasets can be generally separated into two subtasks: (1) the identification of correspondences and (2) the alignment of matching features.

Provided that a matching technique between analogous geometric objects in two distinct datasets exists, one dataset is transformed in such a way that it "fits" with the other. The transformation function considers the geometric differences between the two datasets and the general term rubber-sheeting (cf. Shekhar and Xiong, 2008) is often used for this kind of transformation in which a dataset is compared to a flexible membrane that is elastically force-fitted into a frame and hence obligated to become deformed. Rubber-sheeting can be implemented, for instance, by an interpolation of coordinate differences that are given for control points (Haunert, 2005).

In the context of geodetic and cartographic homogenisation, several methods have been developed, e.g. by Gielsdorf and Gründig (1997), Doytsher et al. (2001). Common to these methods is that they generally carry out planimetric adjustments that move the original coordinates of the geometries in order to align them. Corrections can be applied to the whole dataset, acting as global transformations, or to selected zones only, representing therefore local transformations. These approaches, however, often require that the datasets to be homogenised are in the same scale level (Gründig et al., 2007).

What is required, is a transformation function that handles not planimetric, bur rather height discrepancies between two datasets, and which does not rely on common tie points or same-scale datasets. Another requirement is that the area of influence of such transformation be clearly limited, acting as a local rubber sheeting operation. Although some of existing approaches can be extended to 3D (e.g. Kampshoff, 2005), they still require tie points and modify the original coordinates and are therefore not applicable.

Instead of changing the original coordinates, another common solution is to leave the original data untouched, geometric homogenisation is performed at query level and a rubber-sheeting technique is generally used (Laurini, 1998). If a proper "elastic band" is defined along the common boundary to be transformed, this band represents a transition zone between the two datasets. The transformation should progressively take place inside it, from the external limits of the band toward its middle, so that there is a seamless transition to the original dataset along the external limits of the band. The objects to be warped can be 2D (lines, polygons), but they can also be, for example, the height differences between two neighbouring DTMs.

When it comes to the overlapping zones between two adjacent datasets, a few aspects that will play a role in the following must be underlined: (a) The co-registration algorithms represent the optimal solution to rotation, translation and scaling between two datasets. Nevertheless, they are not necessarily exact, local differences in the overlapping zones can be still found;

(b) Data density in the overlapping zones can be higher than in the input models. Even if this is not necessarily a disadvantage, it represents a type of discontinuity and it might successively lead to problems requiring some sort of local data decimation (Agugiaro et al., 2008);

(c) Decisions regarding the dimension of the overlapping zone are generally tied to rules of thumb ("the larger, the better"). Application-specific rules of thumb exist when it comes to the size of the transition zone. However, an omni-comprehensive, general approach has not yet been presented. In some cases, the extents of the elastic band are set by the user by means of a visual interface. Alternatively, a common, fixed value for the size of transition zone can be defined with no rules other than a certain offset distance from the boundaries.

\subsection{Geometric integration of DTMs}

Given that this article focuses on the integration of surface models that can also be derived from (or can be locally assimilated into) digital elevation models, some related work, concerning geometric integration of DTMs and from which the present approach has taken inspiration, will now be presented.

In general, little literature seems to exist that deals specifically with functions realising elastic deformations applied to digital terrain models. In such cases, the height values of a digital terrain model need to be properly warped in order to guarantee a sufficiently smooth transition.

While describing how to obtain a DTM from patches of different local DTMs, Felus and Csathó (2000) opt for a $1 \mathrm{~km}$ overlap in order to perform the merging operation. Their explanation is that the chosen value is about ten times bigger than the biggest height difference between the two datasets.

Warriner and Mandlburger (2005) discuss how to merge two DTMs with similar quality levels. These must be blended in such a way that there is a continuous transition from one model to the other. In order to obtain a smooth transition, the authors define a tolerance band of constant width. Height values inside the band are taken from both DTMs and a weighted average is performed inside it. The weights depend on the distance from the centreline of the tolerance band. The weight $w_{1}$ for the first DTM gradually increases from 0 to 1, moving from the outer border to the inner border of the tolerance band. In a similar way, the complementary weight is defined $w_{2}=1-w_{1}$.

Katzil and Doytsher (2005) adopt a conceptually similar approach to integrate two overlapping DTMs, assuming the existence of a seam line constructed by using a set of homologous point pairs between the two DTMs. A rubber band is constructed around the seam line and a local geometric correction is performed by morphing the rubber band of each of the adjacent DTMs to the seam line on the merged DTM.

Another approach is presented by Latham and Burns (2006). Given a set of precise objects (called targets), their goal is to smoothly fit the terrain in order to avoid targets floating above the terrain surface. This is achieved by means of a Gaussian correction function over the whole terrain database that corrects terrain height values according to the gap existing between a target and the terrain surface. In this way, the influence of the terrain distortion is planimetrically not delimited, although, in practice, the correction terms become - as desired - negligible with the distance from the targets.

With regards to those approaches that implement warping functions, some questions still remain unanswered: Warriner and Mandlburger's method is designed for two DTMs of analogous 
quality level. Moreover, the transition zone has a fixed, regular shape. What happens if qualities vary or if the tolerance band width varies? What happens if in the approach suggested by Katzil and Doytsher the seam line cannot be constructed, e.g. due to the lack of homologous points? What happens if a second dataset contains objects other than a DTM? Latham and Burns' solution warps the surrounding terrain by means of a Gaussian function; however, its drawback is that the influence of the terrain distortion is not precisely delimited.

The method developed in this article has been inspired from both Warriner and Mandlburger's and Latham and Burns' approaches, however, it overcomes their limitations in four ways: (1) the tolerance band can vary in size and shape, (2) the high resolution dataset must not necessarily be another dataset of analogous quality, (3) the influence of the terrain distortion is clearly delimited and (4) data density inside the transition zone is considered.

\section{Transition surface}

This section represents the main contribution of this article. Section 3.1 presents the prerequisites and Section 3.2 provides a detailed description of the collar's characteristics. Bearing in mind the idea of the elastic zone between neighbouring datasets, the goal is the integration between a low resolution surface model and high resolution surface model. In this process, a proper transition surface and its underlying warping function are developed and evaluated, which allow for a gradual transition between the two datasets. Three criteria must be satisfied: (a) topological and geometric continuity must be guaranteed, (b) a gradual transition in terms of data density, and (c) the size of the "elastic band" is generalised to be of any size, although its shape is still tied to the horizontal plane. The actual procedure on how to obtain the transition surface is divided into successive steps. They are briefly summarised as follows:

(1) Identification of the overlapping zone between the high and low resolution datasets.

(2) New constrained Delaunay triangulation inside the overlapping zone.

(3) Smoothing of the newly obtained triangular mesh.

(4) Data reduction by progressive simplification of the triangular mesh.

The first three steps are grouped and explained sequentially in Section 3.3, while the fourth and final step about mesh simplification is given a more extensive description in Section 3.4.

\subsection{Prerequisites}

Given two input surface models at different resolutions (they will be identified as "high resolution" and "low resolution" models, respectively), the primary requirement in the methodology is that some extra information has been acquired around the high resolution object, so that its extents are actually larger than strictly needed. This extra information, actually very common in laser scanner point clouds, can be thought as a "collar", or as a planimetric buffer region around the high resolution model. To exemplify this concept, one can think of a 3D model of a building acquired by means of a laser scanner: the model comprises the building itself and also some terrain around the building. Frequently, the collar (i.e. the terrain around the building) is simply trimmed or deleted during the successive point cloud editing phase, but in this case some of these extra data are used for modelling purposes, while the actual high resolution object inside the collar (e.g. the building) is left unchanged.
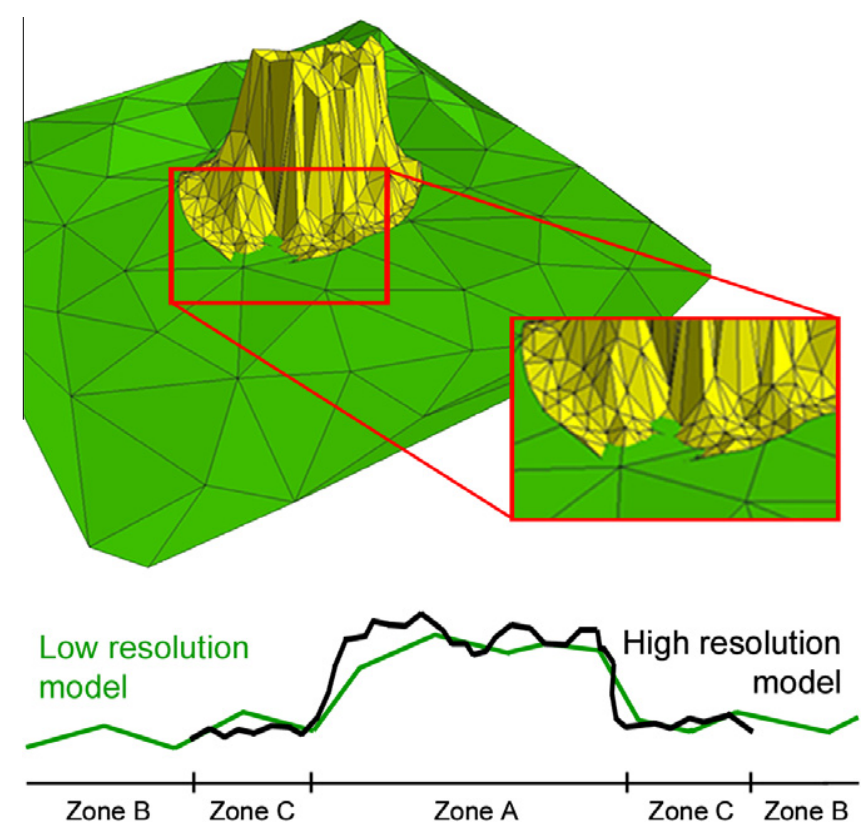

Fig. 1. Geometric and topological inconsistencies between two datasets in which overlaps and gaps exist at the borders of two overlying models: a low resolution model (in green) and a high resolution model (in yellow and black).

The method has three requirements. First, it is assumed that some preliminary data processing has already been carried out on both models so that both datasets consist of triangular meshes without blunders. Irrespective of the original format (e.g. point grid, raster, contour lines, etc.), the data can be converted into a TIN (triangulated irregular network) using standard GIS tools. Second, it is assumed that all datasets share the same coordinate system and have been previously aligned (co-registered) using proper coordinate transformation functions to ensure that the two datasets overlap, at least partially.

Third, it is assumed that both the high resolution data contained in the collar and the low resolution data overlapping the collar are $2.5 \mathrm{D}$, i.e. for each planimetric coordinate, only one height value is given in each model. Elsewhere data is allowed to be also 3D, since it is not directly needed for the integration and modelling purposes.

\subsection{The "collar": identification and properties}

As previously mentioned, fragmented datasets may lead to inconsistencies, e.g. in terms of gaps and overlaps at the borders whenever they cover spatially disjoint regions. Fig. 1 shows two synthetic surface models: a low resolution model (in green colour) and a high resolution object (in yellow and black colours). The two triangular meshes share the same coordinate system and the same feature representations (triangular faces), but the transition from one dataset to the other is not correct due to geometric errors (triangle intersections) and topological discontinuities (gaps). The result is that the resulting model is not an orientable two-manifold unique surface (the desired result). Instead, three zones are generated when the two models are overlapped:

- Zone A: the high resolution object per se. Here the low resolution data refer to a portion of the model which is not to be included in the final, integrated model. The low resolution data in this zone will be eventually discarded;

- Zone B: the outer, "far away" low resolution model features that are not overlapped by any high resolution data. They will remain the same in the final model; 
- Zone C: the overlapping zones, containing data from both high and low resolution models. Points in this zone refer to the same object (the collar); however, some slight differences can be seen with regards to the height profile.

The methodology presented in this article focuses mainly on zone C, which can also be referred to as the "elastic band" zone where the new connecting surface is to be calculated. It is delimited by two borders. The inner border joins the high resolution model to the collar, thus to the transition surface. The inner border is a closed 3D polyline obtained from the adjacent triangle edges of the high resolution mesh. It separates the high resolution object from the collar and is intended to remain unchanged. Similarly, the outer border connects the surrounding low resolution model to the transition surface. It is a closed 3D polyline that originates from the high resolution mesh (it is the outer limit of the collar) and is then projected on the $z$-axis onto the low resolution mesh. Both polylines delimiting the collar are given as input or they can be interactively selected by the user. No other requirements are necessary at this stage, for example, there is no need to input information about mesh segmentation or classification or accuracy values of the surveyed points, etc. Basically, only the 3D coordinates of the mesh nodes, their topological relations to the neighbours and the extents of the collar are known.

For the method to work, the transition surface inside zone $\mathrm{C}$ must: (1) connect both models topologically and geometrically, (2) reflect the characteristics of both datasets and, (3) allow for a progressive transition between the models. Both high and low resolution data in zone $C$ can be used. As a result, the transition surface will be itself a triangulation, having as extents the inner and the outer borders. No changes are made to the height values of the surrounding low resolution model and of the high resolution object (i.e. nothing changes outside the borders). To prevent local height modifications and avoid influencing areas distant from the transition surface, large low resolution triangles not falling completely inside zone $\mathrm{C}$ must be split. Triangles of the transition surface near the inner border must be "closer" to those of the high resolution dataset inside zone $\mathrm{C}$, and triangles of the transition surface near the outer border must be "closer" to those of the low resolution dataset inside zone C. The term "closer" here refers to different properties such as height, slope, and point density, and the result of getting closer to the borders is that the triangles will become more similar to the triangles of either border.

Speaking of height, gaps must be closed at the borders, in other words there must be height continuity (in the following referred as condition (0). In terms of slope, at the borders there must be a smooth transition between the transition surface and the high and low resolution datasets. Along the outer border, triangles of the transition surface should be similarly sloped as those low resolution triangles lying inside zone C. Similarly, along the inner border, triangles of the transition surface should be similarly sloped as those high resolution triangles lying inside zone $C$.

Finally, a transition in terms of point density is required: triangles of growing size should characterise the surface while moving from the inner border to the outer border. This is illustrated in Fig. 2.

\subsection{Steps 1-3: overlapping zone, new triangulation, and smoothing}

The procedure to generate the transition surface consists of four successive steps. The first three steps, described in this section, are:

(1) Identification of the overlapping zone between the high and low resolution datasets, i.e. projection of inner and outer borders onto the $x y$-plane, definition of the annular-shaped domain $\mathrm{A}$, and import of high and low resolution data,

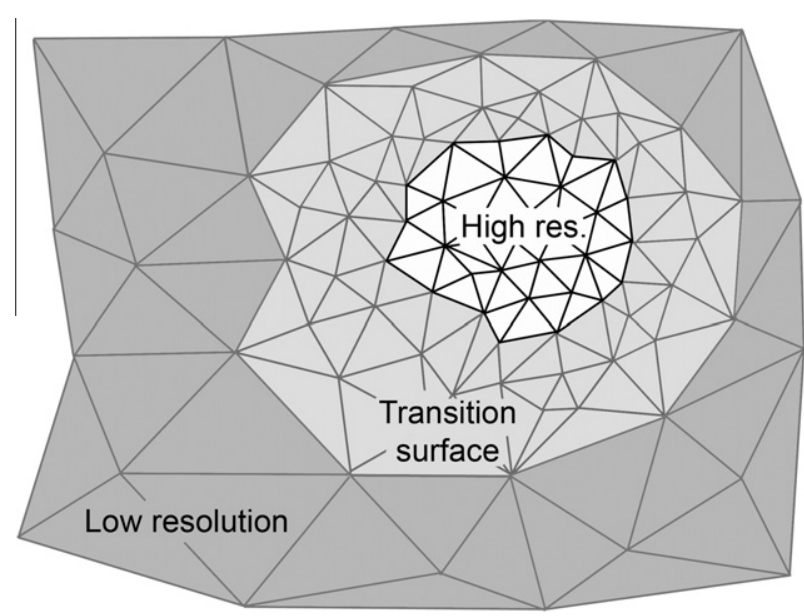

Fig. 2. Conceptual sketch of the transition surface (in light grey): a transition is sought also in terms of point density, i.e. the size of the triangles should increase from the inner high resolution dataset (in white) to the outer low resolution model (in dark grey).

(2) new constrained Delaunay triangulation inside the overlapping zone,

(3) smoothing of the newly obtained triangular mesh by means of spline-based height interpolation model.

In the first step, an initial selection of the data needed for the modelling purposes is carried out and the inner and outer borders are projected onto the $x y$-plane, thus defining a horizontal annularshaped domain A. Domain A is annular because it is topologically equivalent, or homeomorphic, to the mathematical annulus - in this case, a ring-shaped geometric figure in which the outer border represents the outer ring and the inner border represents the inner ring. This domain defines the planar spatial extents that are used to import the necessary data from both the high and low resolution datasets. All high and low resolution triangles that do not lie within the above mentioned zone $\mathrm{C}$ or are not intersected by the inner or outer borders are ignored, thus only a small number of triangles is imported. From this point in the article and unless differently stated, only the imported data will be considered and referred to as the high and low resolution data.

High resolution points (the triangle vertices) are then vertically projected, along the $z$-axis, onto the low resolution triangular faces and vice versa; the local height difference $\Delta z=z_{\text {highres }}-z_{\text {lowres }}$ between the meshes is calculated and stored on a per point basis.

In the second step, in order to close the gaps and to permit $\mathrm{CO}$ continuity between adjacent triangles on both sides of the borders, a new constrained Delaunay triangulation is calculated, the borders are treated as break lines. The external vertices of overlapping low resolution triangles are also included in the Delaunay triangulation, in order to generate a topologically continuous surface in which the outer border points are now connected to the low resolution ones. Large triangles, falling partially inside and partially outside the overlapping zone $C$, are split. No other constraints are considered at this stage. This process is illustrated later on in Fig. 6 where on the left image the height profile in the transition zone is not smoothed, although the two meshes are now correctly merged. The right image shows instead a smoothed height profile.

In the third step, the height profile inside the transition surface must be smoothed so that the height values inside zone $C$ are more "similar" to the low resolution triangles that are closer to the outer border, and - vice versa - the height values closer to the inner border are more "similar" to the high resolution triangles. This means 
that, inside the overlapping zone, every point is required to have an intermediate height value $z_{\text {new }}$ given by

$z_{\text {new }}=(1-\lambda) z_{\text {lowres }}+\lambda z_{\text {highres }}=z_{\text {lowres }}+\lambda \Delta z$

with $z_{\text {lowres }}$ : height value in the low resolution mesh (for high resolution points, the projected height value is used), $\Delta z=z_{\text {highres }}$ $-z_{\text {lowres }}$ : point-wise vertical distance between the two meshes, $\lambda=\lambda\left(d_{n}\right)$ : a weight function depending on a distance parameter $d_{n}$, which will be defined in the following (see Eq. (4)).

Therefore, a proper weight function $\lambda=f\left(d_{n}\right):[0,1] \rightarrow[0,1]$ needs to be formulated. It depends on a yet-to-be-defined distance parameter $d_{n}$ (normalised distance from the outer border, see below) and maps the value of the weight value $\lambda\left(d_{n}\right)$ according to the distance from the outer and the inner border (they correspond to $d_{n}=0$ and $d_{n}=1$, respectively). Let's assume that $\lambda\left(d_{n}\right)=0=$ const $\forall d_{n} \in[0,1]$, then all triangle vertices of the transition surface would lie on the low resolution mesh. If instead $\lambda\left(d_{n}\right)=1=$ const $\forall d_{n} \in[0,1]$ is assumed, they would all lie on the high resolution mesh. Either case could eventually lead to a step discontinuity at the borders because no gradual transition would be made.

In order to shape a gradual transition in terms of height profile inside the transition surface, different weight functions can be modelled. For example, the simplest, linear function $\lambda=d_{n}$ (cf. Warriner and Mandlburger, 2005) or an exponential function $\lambda=d_{n}^{a}$ (with $a>0, a \neq 1$ ) represent possible solutions; however, these functions do not yield the desired smoothing effect at both borders when applied to the height differences in the transition surface (see Eq. (1)). Instead, what is required is a weight function that obeys the following conditions:

$\begin{cases}f(0)=0 \wedge \lim _{d_{n} \rightarrow 0+} f^{\prime}\left(d_{n}\right)=0 & \text { (outer border) } \\ f(1)=1 \wedge \lim _{d_{n} \rightarrow 1-} f^{\prime}\left(d_{n}\right)=0 & \text { (inner border) }\end{cases}$

A piecewise polynomial curve like a uniform spline of order 3 as

$\lambda=f\left(d_{n}\right)=\left\{\begin{array}{cc}2 d_{n}^{2} & 0 \leqslant d_{n} \leqslant 0.5 \\ -2 d_{n}^{2}+4 d_{n}-1 & 0.5<d_{n} \leqslant 1\end{array}\right.$

has been chosen, because it complies with the conditions in Eq. (2). The chosen spline function and the other mentioned functions are plotted in Fig. 3.

As stated before, projecting the inner and outer borders on the $x y$-plane leads to an annular-shaped domain A. When it comes to the distance parameter $d_{n}$, this represents the normalised distance from the outer border, calculated on the $x y$-plane, for any point $P(x, y)$ lying within domain A. In general terms, $d_{n}$ can be thought as a function $d_{n}=g(P): A \rightarrow[0,1]$ and it can be written as follows:

$d_{n}=g(P)=\frac{d_{\text {out }}(P)}{d_{\text {out }}(P)+d_{\text {in }}(P)}$

with $P(x, y)$ : any point belonging to $\mathrm{A}, d_{\text {out }}(P), d_{i n}(P)$ : distance from point $P$ to the outer and inner border, respectively, calculated with regard to the annular topology of domain A.

With regular convex shapes, computation of distance can be generally performed with simple Euclidean distance functions that return the minimum distance between two geometry entities. However, in case of extremely irregular shapes (which may show concavities at the borders, presence of multiple holes, etc.) computation of distance using Euclidean functions can lead to errors. Fig. 4 illustrates some examples. In both pictures, the shortest path from a point to the inner border inside the light grey area is depicted in black, while dark grey indicates the shortest path to the outer border. The circular shape (on left) shows that Euclidean distance functions provide a correct value for regular convex shapes. However, in the right example, (analogous results are represented by solid lines) the results are not always acceptable, as

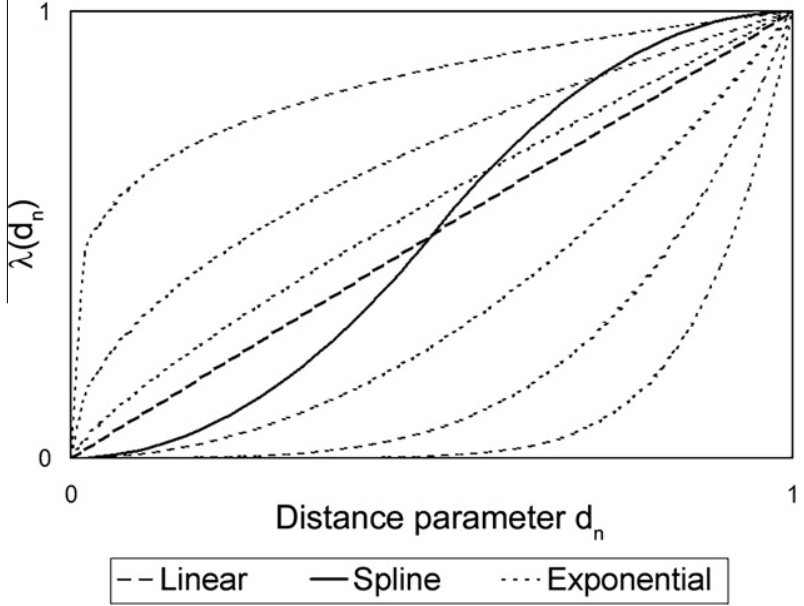

Fig. 3. Different weight functions: linear, exponential (with varying exponential values) and spline.

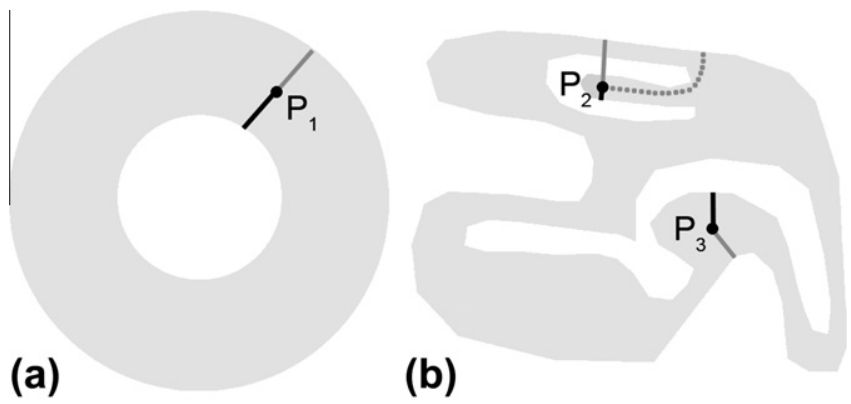

Fig. 4. Inside the overlapping zone, i.e. inside the horizontal annular-shaped domain A (in light grey), standard Euclidean distance functions provide correct results from a point $P_{1}$ to the inner and outer border (in black and dark grey solid line, respectively) if the shape is regular (a). In case of irregular shapes (b) they may deliver incorrect results, because distance is computed across the borders. Correct results for point $\mathrm{P}_{2}$ are sketched by the dashed line.

they may cross the borders, and thus they do not respect the annular topology of the irregular shape. The dashed lines sketch the shortest path lying completely within the orange surface and represent the correct results.

A solution to this problem is to calculate distances from the inner and outer borders separately and successively combine the two partial results. The distance values $d_{\text {out }}(P)$ and $d_{\text {in }}(P)$ are computed through progressive iterative buffering. Once a buffer width is chosen, buffers are gradually calculated from the inner border outwards and vice versa. A combination of the two resulting maps, according to Eq. (4), yields the desired normalised distance $d_{n}$ from for any point in domain A to the outer border, see Fig. 5. Since, now, both the weight function $\lambda\left(d_{n}\right)$ and the relative parameter $d_{n}$ have been defined, Eq. (1) can be applied to any point belonging to the transition surface. An example is shown in Fig. 6 (see right image).

\subsection{Step 4: mesh simplification}

A transition in terms of point density has not yet been achieved - density should decrease gradually, moving on the mesh from the inner to the outer border of the transition surface. To achieve this, simplification, i.e. the process to automatically reduce the complexity of a given model (Luebke et al., 2002), is used. In the fourth and final step, a simplification algorithm that preserves the 2D topology of the surface (no gaps or cracks must be present in the output model) and eliminates existing points from the mesh 
without having to calculate or insert new ones is applied. In addition, the simplification algorithm should also allow users to set a variable error throughout the transition surface, i.e. a smaller one near the high resolution zone and a larger one near the low resolution zone. According to the Nyquist-Shannon sampling theorem, a higher point density results in shorter mutual distances among mesh points (i.e., smaller triangles), allows for a more accurate point-wise sampled surface. On the one hand, all non-sampled points (here: the mesh triangles) are assumed to lie closer to the real, unknown surface. On the other hand, the larger triangles, which can be found in the low resolution model, correspond to lower point densities, so the differences between the mesh and the real surface can be assumed to be potentially bigger than in the previous case. These considerations introduce the need to adopt a variable-error simplification algorithm.

Among the existing approaches in Computer Graphics, the "Simplification envelopes" algorithm (Cohen et al., 1996) fulfils these requirements. The algorithm creates two offset surfaces that are a user-defined $\varepsilon$ value distant from the input triangular mesh. The "outer" surface is created by a displacement along the normal vector of every vertex by $\varepsilon$ and the "inner" surface is created by displacing it by $-\varepsilon$. Given that both envelopes are not allowed to self-intersect, the simplified model surface will lie between the offset surfaces. The $\varepsilon$ value can be variable on a per point basis permitting varying approximation to be achieved, i.e. details that must not be simplified beyond a certain level can be preserved. The $\varepsilon$ parameter defines the "thickness" of the envelopes: the bigger the $\varepsilon$ value is, the thicker the envelopes, and the further the mesh simplification proceeds. Due to the envelopes' geometry, all resulting triangles are no farther than $\varepsilon$ from the original surface. Once the two offset surfaces have been obtained, the actual simplification process starts. Iteratively, a vertex is removed and a hole with $n$ sides is created in the input mesh. A new triangulation is carried out inside the hole, resulting in $n-2$ new triangles. All the vertices of the new triangles already lie within the offset surfaces; however, the triangles must lie within the offset surfaces and must not intersect existing triangles of the simplified surface.
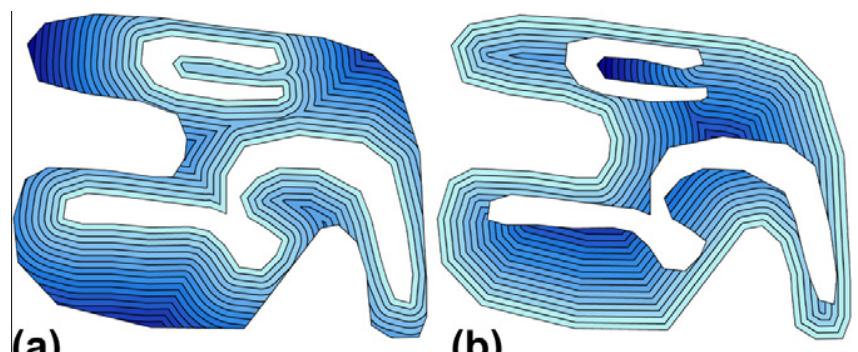

(a)

(b)

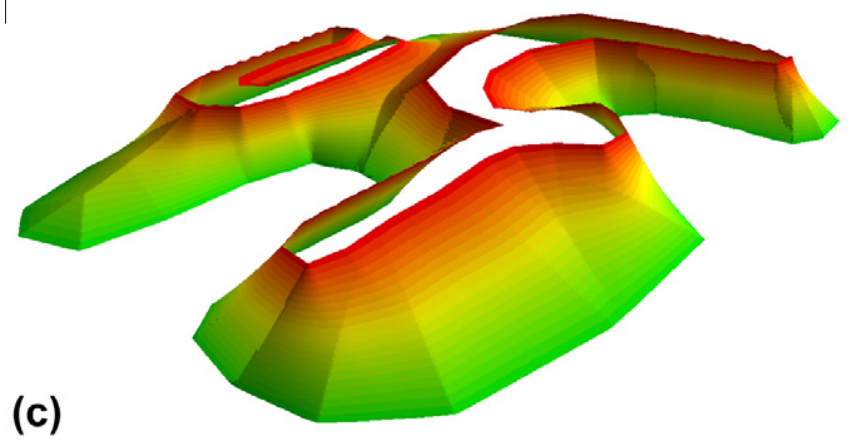

Fig. 5. Example of progressive buffering from the inner borders outwards (a) and vice versa (b), inside an irregularly shaped domain. 3D representation of the combination of the two buffer maps, which yields the normalised distance from the outer border for any point inside the irregular shape (c).

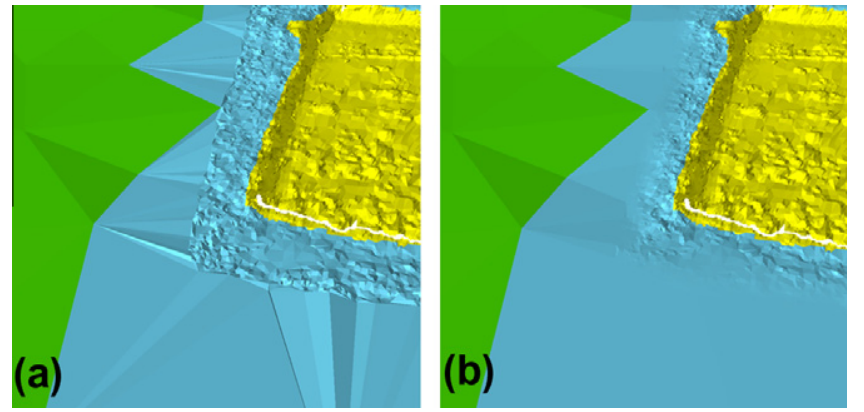

Fig. 6. The area highlighted in blue shows the difference between before (a) and after (b) smoothing the height profile of the transition surface, which connects the low resolution model (in green) to the high resolution model (in yellow).

Every new triangle candidate is tested for intersections with the envelopes and with the surrounding triangles. If all collision tests succeed (i.e. no intersections), then the new triangulation of the hole is accepted and the algorithm proceeds to the next vertex.

The algorithm, as formulated by Cohen et al., is employed to simplify models in the field of Computer Graphics. Its application to the integration of triangular meshes of different resolutions is new, and requires the systematic variation of the $\varepsilon$ value to be functionally determined. In the following, it is shown how the $\varepsilon$ value will be modelled in such a way that it (a) maps the requirements of the transition surface listed in Section 3.2 and (b) complies with the Simplification envelopes algorithm as given by Cohen et al.

Given that the mesh simplification is desired to grow gradually from the inner border to the outer border in the transition surface, a correspondence can be established between $\varepsilon$ and a parameter $d_{n}^{c}$, the latter being the complementary to the distance parameter in Eq. (4), i.e. $d_{n}^{c}=1-d_{n}$. By setting $\varepsilon=0$ at $d_{n}^{c}=0$ for the inner border points and letting it grow toward the outer border up to $\varepsilon_{\max }$ for $d_{n}^{c}=1$, a gradual transition can be modelled in terms of detail reduction. Similarly to the weight function used for the height profile, a distance-dependent $\varepsilon$ function can be shaped, from the inner to the outer border, and is defined as

$\varepsilon=h\left(d_{n}^{c}, \varepsilon_{\max }\right)=f\left(d_{n}^{c}\right) \cdot \varepsilon_{\max }$

with $d_{n}^{c}$ : normalised distance from inner border, $f\left(d_{n}^{c}\right)$ : a spline function, analogous to Eq. (3), $\varepsilon_{\max }$ : maximum displacement value of the offset surfaces.

Fig. 7 offers an example: for a given $\varepsilon_{\max }$, triangles grow gradually in size from the inner border to the outer border (and according to the surface irregularity of the input mesh). In addition, for growing values of $\varepsilon_{\max }$ it is possible to achieve greater simplification levels. In the following, it helps to remember that $\varepsilon_{\max }$ represents an implicit quality parameter for the output mesh, and, more specifically, it characterises the mesh along the outer border, that is at the junction with the low resolution dataset.

\subsubsection{Determination of the proper simplification level}

For the mesh simplification process it is crucial to choose $\varepsilon_{\max }$ properly, because it must relate to the low resolution model into which the transition surface is being embedded. The selection of the $\varepsilon_{\max }$ value depends on a variety of factors like the accuracy and the geometry of the surrounding low resolution model, or, more importantly, on the presence or absence of such information. If nothing is known about accuracy, at least a global value should be assumed on the basis of the surveying technique used for the low resolution model. If this information or similar metadata are also missing, a characterisation can be performed using geometry only. If no suitable $\varepsilon_{\max }$ can be obtained in this way, some 

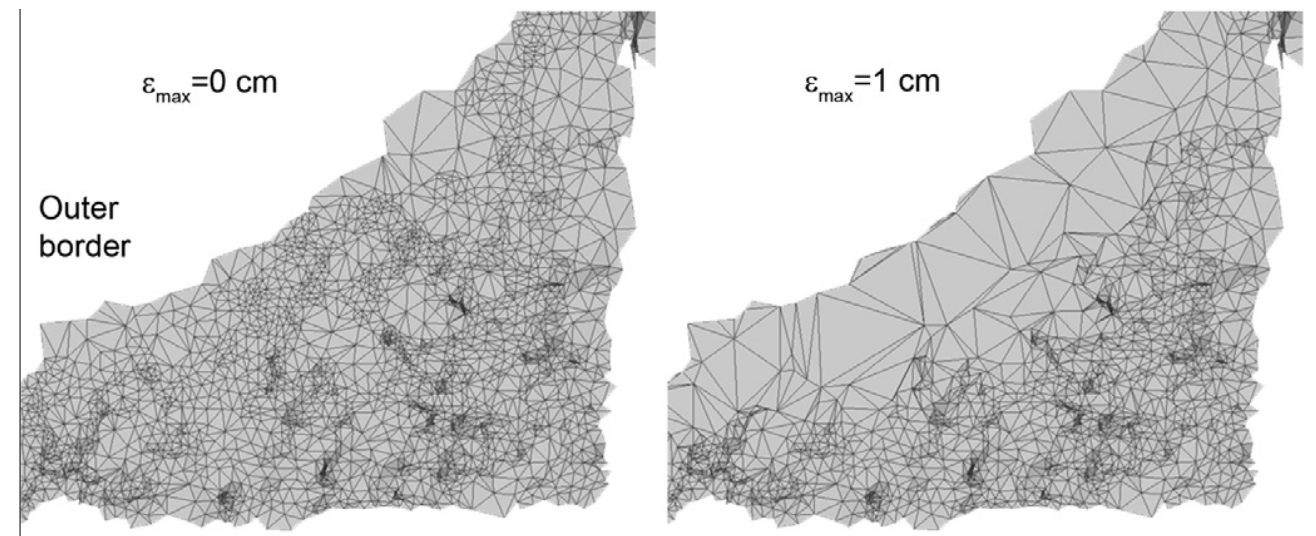

Inner border
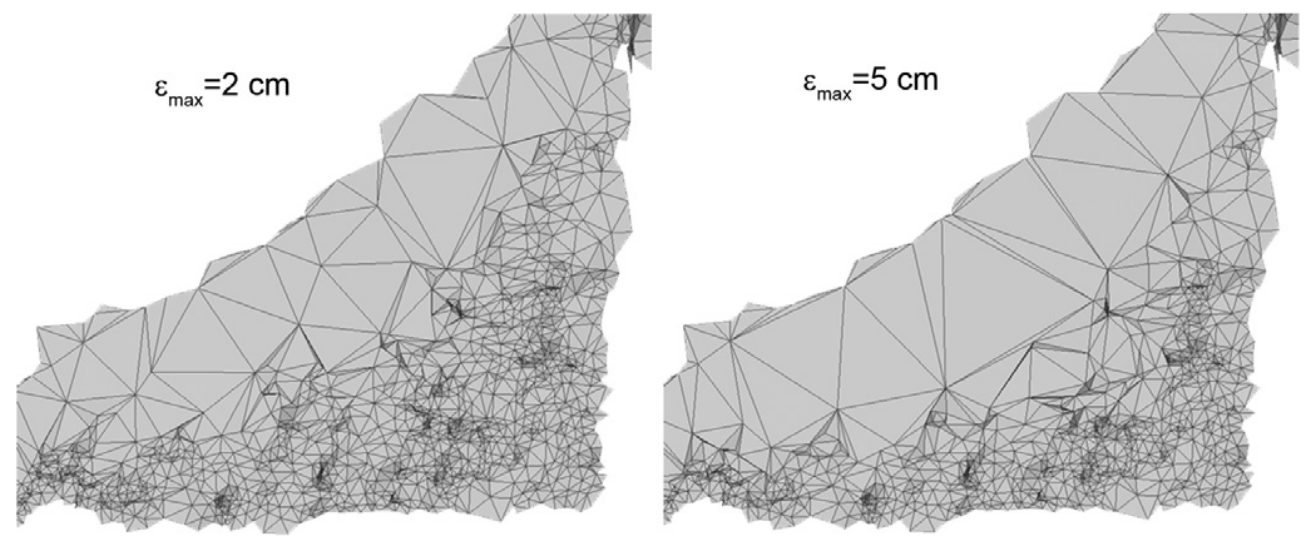

Fig. 7. Progressive simplification in the transition zone, between the inner border and the outer border, with growing values of $\varepsilon_{\text {max }}$.

heuristics should be employed. Some strategies are presented here to set the $\varepsilon_{\max }$ value. Some critical cases and their possible solutions will also be mentioned.

3.4.1.1. $\varepsilon_{\max }$ as maximum displacement error. The first strategy considers $\varepsilon_{\max }$ in terms of maximum error bound for simplification envelopes. In other words, the simplified triangles of the transition surface are no farther than $\varepsilon_{\max }$ from the unsimplified mesh. If a global accuracy value $\sigma_{H}$ for the low resolution points is known (and often this is the only one known), a first solution consists in setting $\varepsilon_{\max }=\sigma_{H}$. In a conceptually analogous way to simplification envelopes, every low resolution triangle is thought to be contained between an upper and a lower "enveloping" triangle, which is vertically displaced by $\sigma_{H}$. The 3D displacement vector can thus be written as $\vec{\varepsilon}_{D T M}=\left(0,0, \sigma_{H}\right)$. Similarly, the 3D displacement vector for the transition surface on the outer border can be written as $\vec{\varepsilon}_{O . B .}=\left(\varepsilon_{x}, \varepsilon_{y}, \varepsilon_{z}\right)$. Setting $\varepsilon_{\max }=\left\|\vec{\varepsilon}_{O . B .}\right\|=\left\|\vec{\varepsilon}_{D T M}\right\|=\sigma_{H}$ is therefore an approximate but conservative approach, since $\varepsilon_{z}=\varepsilon_{\max }$ co$\mathrm{s} \alpha \leqslant \sigma_{H}$ (with $\alpha$ angle between the normal vector and the $z$-axis) and $\varepsilon_{z}=\sigma_{H}$ only for $\alpha=0$, i.e. only when $\vec{\varepsilon}_{0 . B \text {. }}$ is itself vertical and oriented upwards. The first experimental example in Section 5 adopts this strategy.

3.4.1.2. $\varepsilon_{\max }$ as mesh irregularity parameter. With no information about the accuracy of the low resolution model, a second strategy consists in defining $\varepsilon_{\max }$ on the basis of the low resolution mesh irregularity. A quick mention to the Douglas-Peucker algorithm must be made here: given an $\varepsilon_{D P}$ error parameter, the DouglasPeucker algorithm outputs a simplified, smoothed polyline curve that is no farther than $\varepsilon_{D P}$ from the original polyline. However, if the idea behind this algorithm is somehow "inverted", a minimum
$\varepsilon_{D P}^{r}>0$ can be calculated from a previously smoothed polyline. For any value in $\left[0, \varepsilon_{D P}^{r}\right]$ no smoothing takes place, however the polyline can be further simplified for values greater than $\varepsilon_{D P}^{r}$ (the critical cases in which $\varepsilon_{D P}^{r}=0$ or $\varepsilon_{D P}^{r}$ cannot be obtained will be discussed later). In an analogous way, the low resolution triangulation can be thought as the result of a previous (unknown) simplification process, so if an $\varepsilon_{D P}^{r}>0$ exists, then it can be used as the geometric connecting value between the low resolution model and transition surface by setting $\varepsilon_{\max }=\varepsilon_{D P}^{r}$.

In order to obtain the $\varepsilon_{D P}^{r}$ value, the simplification envelopes algorithm is iteratively applied to the low resolution triangles falling inside or intersecting zone C. A variable, growing $\varepsilon_{\max }$ is used, starting from a positive $\varepsilon_{\max } \approx 0$, until the first simplification takes place, i.e. the number of low resolution triangles is reduced. The second experimental example in Section 5 adopts this strategy.

3.4.1.3. Heuristic approach to determine $\varepsilon_{\max }$. Finally, if the previous two approaches fail, then some heuristic workarounds can be employed. However, some considerations about the simplification algorithm are necessary at this point. The currently implemented algorithm removes all mesh vertices until the remaining triangles cannot be further simplified without intersecting the offset surfaces. As a matter of fact, the algorithm acts as low-pass filter preserving the low frequency variations of a surface, while the high frequency ones are smoothed. Point reduction is thus dependent only on the irregularity of the surface. This means that over-sampled datasets can be simplified without introducing any error in the output geometry. Theoretically, one of the strengths of triangular meshes (e.g. a TIN-based DTM) is that plane areas with no height changes can be represented with few large triangles, while smaller triangles are used for more irregular areas. This phenomenon, however, is not 
always true in real-world models: a certain amount of redundant data may be still present. As a consequence, the second strategy with an over-sampled low resolution dataset can lead to $\varepsilon_{\max }=\varepsilon_{D P}^{r}=0$ and to no data reduction inside the transition surface (due to the simplification not starting). Although this critical case remains so far without solution, it represents an extreme situation, because, with real data coming from surveying techniques, some operational margin is indeed left and leads to a certain positive $\varepsilon_{\max } \neq 0$. The heuristic workaround relies on an iterative approach, similar to the previous one: multiple simplified transition surfaces are obtained starting from the same input mesh, but with growing values of $\varepsilon_{\max }$. The number of remaining triangles after each simplification is plotted against the growing $\varepsilon_{\max }$ values, the resulting curve is called "simplification curve". Fig. 12b provides, later on in the article, an example of a "simplification curve".

According to the experimental tests done on real datasets, a value of $\varepsilon_{\max }$ has been empirically found to deliver acceptable results if it is selected from the interval $0 \leqslant \varepsilon_{\max } \leqslant \bar{\varepsilon}_{\max }$, with $\bar{\varepsilon}_{\max }$ representing the value where the simplification curve assumes a relatively linear trend. In the example in Fig. 12b, this point is highlighted with a circle. If the iterative process is matched with a continuous visual inspection of the resulting simplified meshes, this operation can be, of course, of great help to select the $\bar{\varepsilon}_{\max }$ value. The third and final experimental example in Section 5 adopts this strategy.

A second critical case originates when the $\varepsilon_{D P}^{r}$ value cannot be obtained. In the case of a polyline, this means that the curve has been previously smoothed to such an extent that it is actually a single line segment (thus a 1-simplex), which cannot be further simplified, regardless of $\varepsilon_{D P}^{r}$. Extending the concept to the low resolution model, this corresponds to embedding the transition surface into a single, large triangle (thus, a 2-simplex). In order to overcome this drawback, a possible solution is to import at least the neighbouring triangles and then calculate the $\varepsilon_{D P}^{r}$ value using the above mentioned strategies. However, this workaround breaks the initial assumptions regarding the datasets to be integrated that argue to use only information from the area surrounding the transition zone, and it might be problematic to obtain a mesh irregularity parameter from adjacent, but distant triangles that are not directly related to the overlapping zone. A possible solution to these critical cases will be discussed in the last section with regards to future improvements.

\section{Implementation}

The methodology presented in this article has been implemented using nearly exclusively free and open-source software. PostgreSQL 8.2 has been chosen as the object-relational database, together with PostGIS 1.3, which adds support for geographic objects. It should be noted that PostGIS has a topology support that consists in a schema model and functions to handle topological element faces, edges and nodes; it is, however, still in the developmental stage (as of February 2012). Database server functionality has been extended with the built-in procedural languages PL/ pgSQL, which allows scripting inside the server, and permits to join the power of a procedural language with SQL. For mesh inspection and light editing tasks, MeshLab was used: it is a free and opensource extensible platform to process and edit 3D triangular meshes. Advanced mesh editing was carried out using RapidForm 2004 by Inus Technology. For the constrained triangulation tasks, the free and open-source TRIANGLE software by Shewchuk (1996) was employed, while the mesh simplification was performed using the freely available version of "Simplification envelopes" by Cohen et al. (1996). Quantum GIS was used for the visualisation and inspection tool because of its support for the PostgreSQL/PostGIS datasets and is easily integrated with GRASS GIS (used for 3D views on GNU/Linux systems), while the ESRI ArcGIS suite (i.e. ArcScene) was employed for 3D visualisations on Microsoft Windows systems.

For data import and export from and to PostgreSQL, the PLY file format, also known as Polygon File Format, has been adopted. It is a well-known, documented and open format designed to store threedimensional data from 3D scanners. It allows users to store the polygons of a mesh together with optional attributes for the vertices or for the faces. Finally, it is one of the supported input/output formats of MeshLab and the standard file format for "Simplification envelopes". The two input PLY files, one for the high resolution model and one for the low resolution model and each containing the vertex coordinates and the face topology, were imported separately into two distinct PostgreSQL database schemas (namely "hr_model" and "lr_model"). For each schema, data were saved into a "points" and a "faces" table. A third schema called "model" was also created, where most of the data processing took place and all the needed tables, views and developed SQL functions were contained. This three-schema-structure was intended to keep the original datasets separated from each other and from the to-be-calculated transition surface. Moreover, it was meant to ideally simulate two distinct data sources that might be accessed remotely. More details about implementation can be found in Agugiaro (2009).

\section{Experimental results}

This section presents the experimental results, carried out using data from real-world models. All three methods to determine $\varepsilon_{\max }$ (described in Section 3.4.1) were used. In each experiment, the method selected depended on the quality of the data and the quantity of accompanying information. Three examples of data integration were evaluated to explore the methodology's potential in different applications with data of varying size and characteristics. These examples are: 3D city modelling, archaeological excavations and small cultural heritage objects. Additional tests have been successfully conducted in the context of cultural heritage using the three methods presented here (Agugiaro, 2009; Agugiaro and Kolbe, 2009).

A standard colour-coding is used to simplify the identification of all models: the low resolution mesh is coloured in green, the transition surface in blue and the high resolution mesh (if present) in yellow. All meshes are triangular, they have already been previously aligned (i.e. co-registered) and are topologically sound, i.e. no mesh self-intersections, no fold-overs, etc.

The first example deals with the integration of an urban object into a surrounding DTM. The laser-scanner-acquired point cloud of an underground entrance (U2 metro station at Ernst-Reuter-Platz in Berlin) was embedded into the triangulated low resolution TIN-based DTM, whose global vertical accuracy value is known from the data acquisition process $\left(\sigma_{H} \approx 10 \mathrm{~cm}\right)$. The chosen strategy to set the $\varepsilon_{\max }$ at the outer border is, therefore, $\varepsilon_{\max }=\sigma_{H}$ (cf. Section 3.4.1.1). Both models are shown overlapped in Fig. 8a, while in Fig. 8b-d only the collar is shown (the high resolution object, i.e. the railing, the stairs and the initial part of the tunnel, is hidden for a better visualisation). The procedure steps explained in the previous sections are quickly mentioned in the accompanying caption.

The datasets used in the second example refer to a portion of the archaeological site located in Nora, Sardinia, Italy (Bejor, 1992). In this example, the objective is to embed the high resolution mesh with architectural elements of a temple into the coarser 

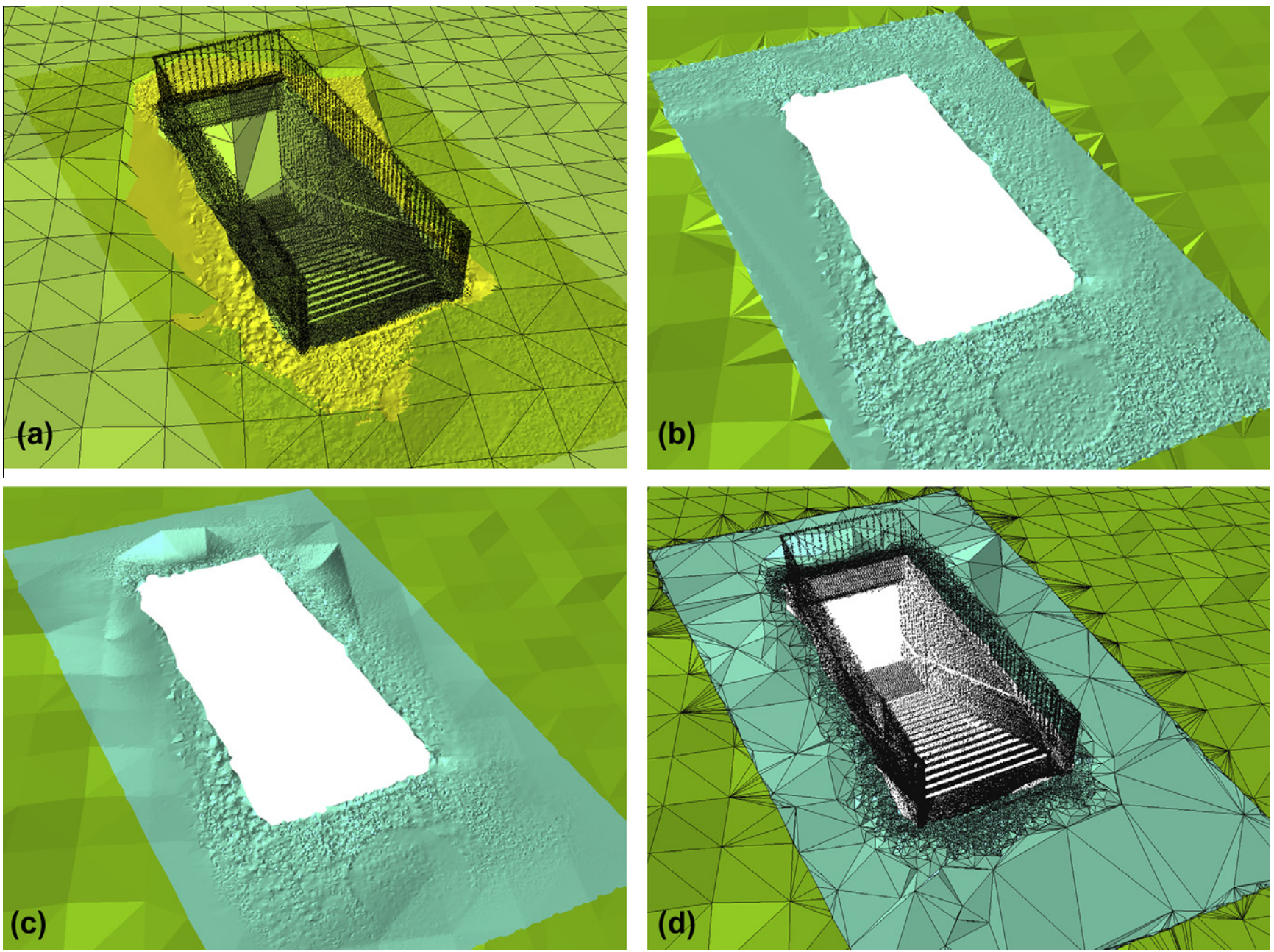

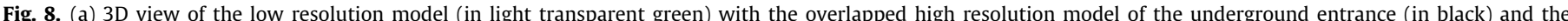

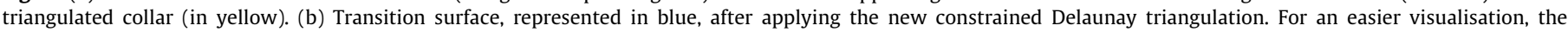

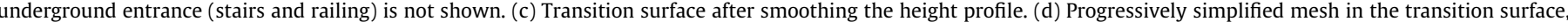

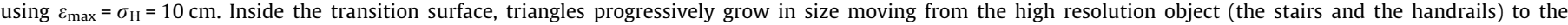
surrounding terrain model.
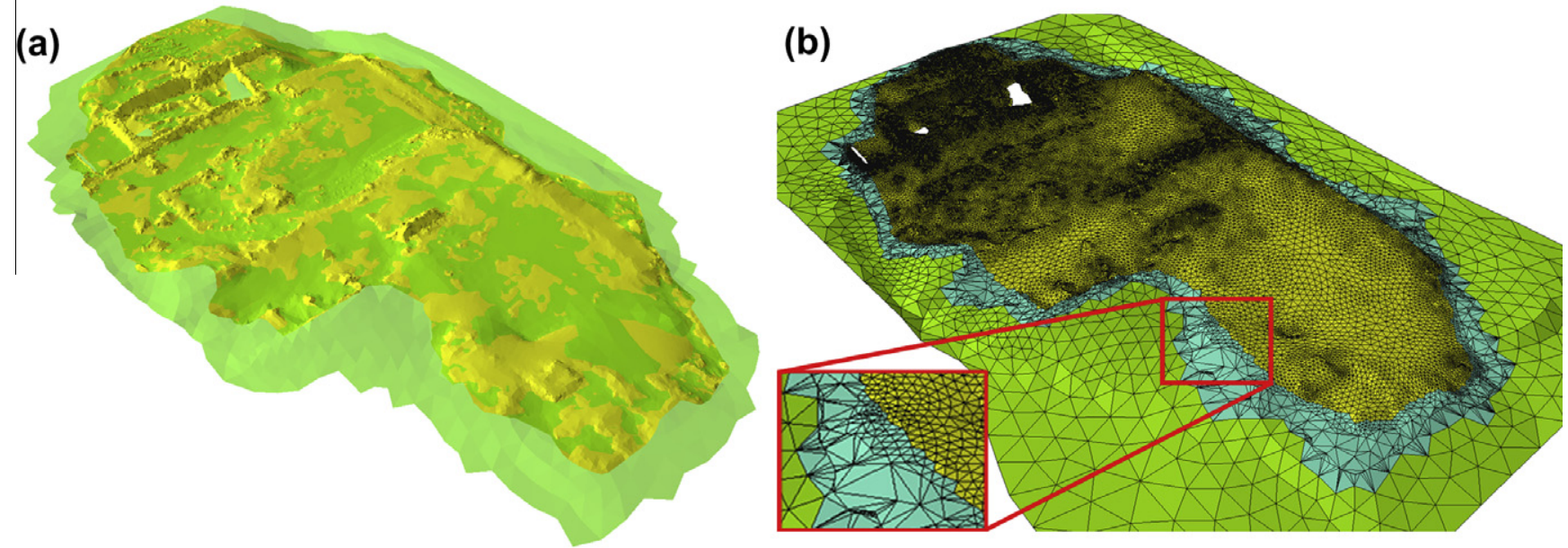

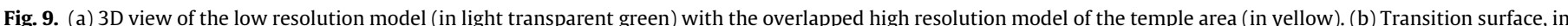
blue, obtained by setting $\varepsilon_{\max }=1 \mathrm{~cm}$ for the mesh simplification. It connects the low resolution model (in green) to the high resolution model (in yellow). 


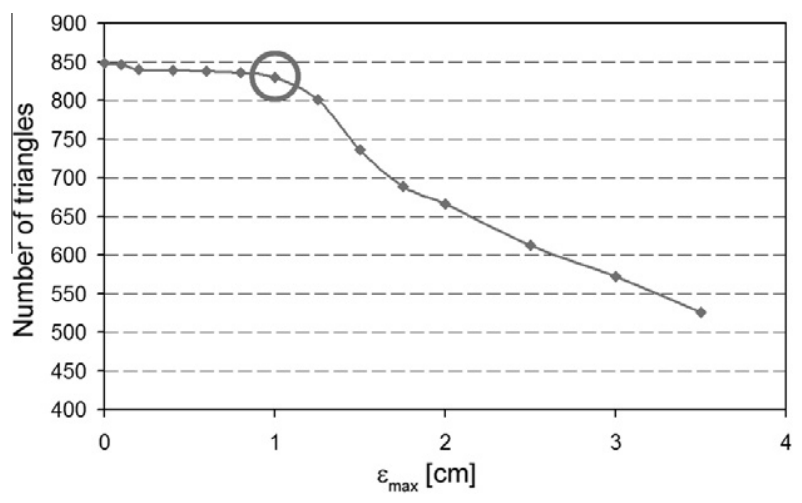

Fig. 10. Simplification curve of the imported low resolution dataset. The threshold is chosen at $\varepsilon_{\max }=1 \mathrm{~cm}$

mesh representing the terrain. In this example, the second, geometrical strategy is chosen (cf. Section 3.4.1.2), i.e. a simplification curve is obtained from the low resolution triangles used for the transition surface. The two overlapping datasets are presented in Fig. 9a, while in Fig. 9b the resulting transition surface, connecting both input dataset, is represented in blue. The simplification curve is obtained from the low resolution triangles used for the transition surface, see Fig. 10. An $\varepsilon_{D P}^{r}$ is identified and subsequently set to $\varepsilon_{\max }=\varepsilon_{D P}^{r}=1 \mathrm{~cm}$. Again, the procedure steps explained before are briefly mentioned in the accompanying captions.

The last example is intended to test how details are preserved in the transition surface. The datasets used in this example are from a family emblem of the Scaliger tombs - a group of five Gothic funerary monuments celebrating the Scaliger family, who ruled Verona, Italy, in the 13th-14th century (Fabris et al., 2011). This example simulates a common problem in the framework of cultural heritage. That is, a particularly elaborated decoration might have to be embedded into the surrounding lower resolution model. From the original laser-scanner-acquired model, a dominant plane can be identified and used as a $x y$-plane after a coordinate transformation. In this case, two models have been taken: (1) a low resolution one has been obtained by point decimation, and (2) for the high resolution model, the eagle detail has been cut out, the intention being to embed it into the low resolution surface. The two models are represented in Fig. 11a, the transition surface connecting them is represented in Fig. 11b, as well as the final integrated model in Fig. 11c. In this example, the third, heuristic approach has been chosen to set the $\varepsilon_{\max }$ value (cf. Section 3.4.1.3), because no "step" can be clearly seen in the green simplification curve of Fig. 12a. Therefore, a simplification curve has been obtained from the transition surface and is shown in Fig. 12b. The value $\bar{\varepsilon}_{\max }=10 \mathrm{~mm}$ was chosen as the upper bound of the interval [0, $\left.\bar{\varepsilon}_{\max }\right]$. The $\varepsilon_{\max }$ value for the transition surface was $\varepsilon_{\max }=6 \mathrm{~mm}$.

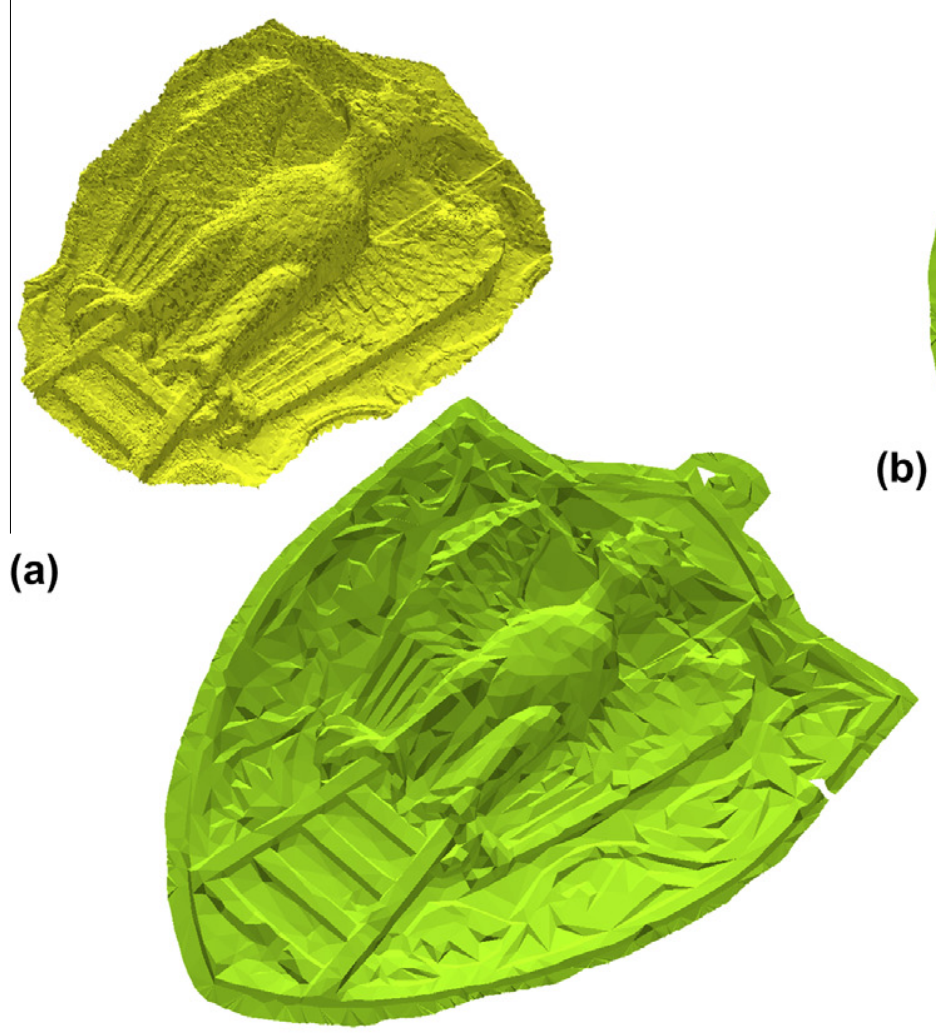

(c)

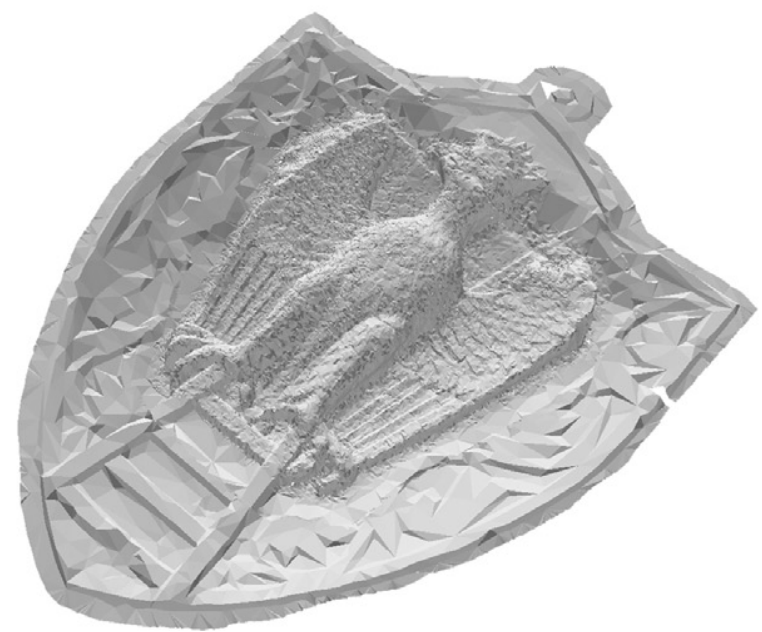

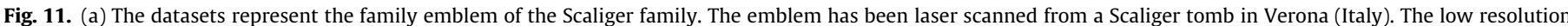

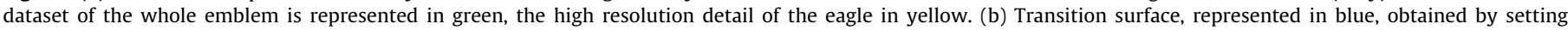

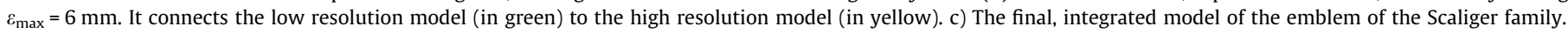



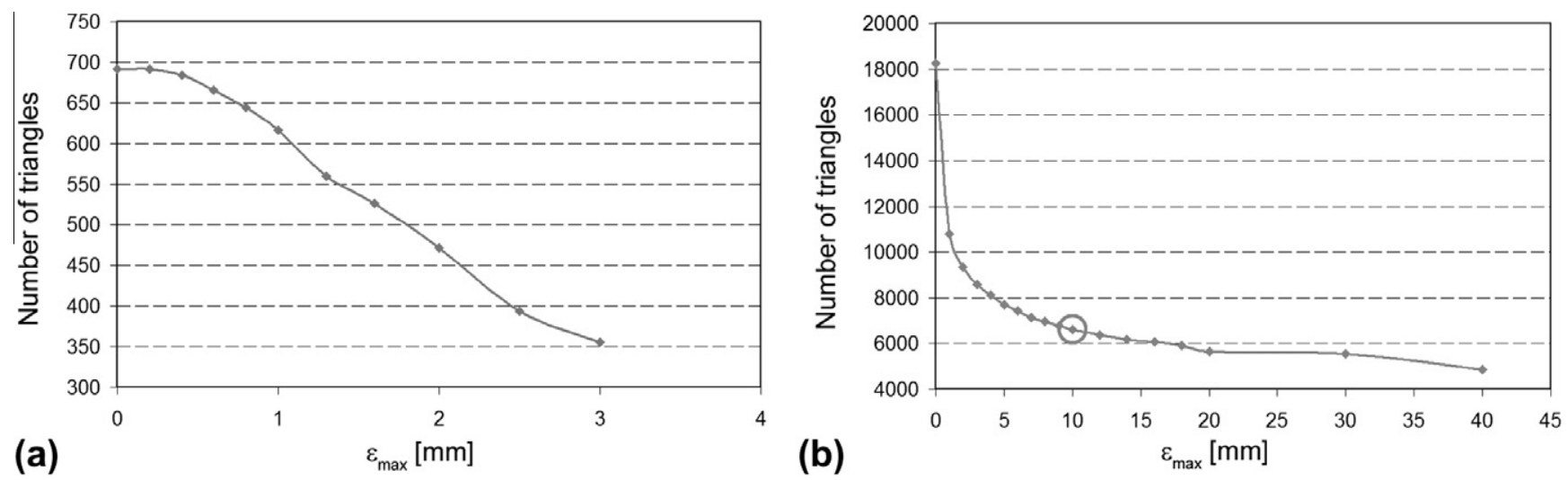

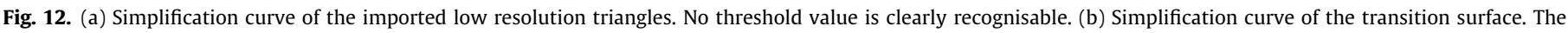
interval for the $\varepsilon_{\max }$ is $0 \leqslant \varepsilon_{\max } \leqslant \bar{\varepsilon}_{\max }$. Value $\bar{\varepsilon}_{\max }=10 \mathrm{~mm}$ is highlighted on the curve.

\section{Conclusions}

This article has presented a data integration method for triangular-mesh-based surface models of different resolutions. Given two previously aligned models, which consist of triangular meshes at different resolutions, some extra geometrical information is required around the high resolution object, as a sort of planimetric buffer, here called "collar". In addition, it is required that both the high resolution data contained in the collar and low resolution data overlapping the collar are 2.5D.

The developed method takes into account different quality aspects of the low and high resolution models. These quality aspects are smoothly interpolated while moving from one model to the other by means of a transition surface. In order to obtain the transition surface, the procedure has been divided into four successive steps. First, only the necessary high and low resolution data needed for the modelling purposes are selected from the input datasets: only those high resolution triangles forming the collar and the low resolution triangles overlapping it are imported, while all remaining data are ignored. Second, a new constrained Delaunay triangulation is computed inside the overlapping zone, in order to guarantee topological continuity between the two models. Third, the height profile of the triangular mesh in the transition surface is smoothed by means of a spline-based interpolation function, and fourth, a progressive mesh simplification takes place to reduce data selectively.

Results from the experimental tests show that, using the proposed methodology, a suitable transition surface can be created, and the initial continuity requirements in terms of topology, geometry and point density can be fulfilled; in the overlapping zone (the so-called zone $C$ ), the transition surface uses information from both datasets and allows for a gradual transition between them, both in terms of height and slope. Moreover, a progressive mesh simplification is also achieved. The resultant transition surface permits, therefore, a local update of a low resolution model through a geometrically and topologically correct insertion of a high resolution model, provided that the requirements to the input models are respected.

The methodology was developed keeping in mind the problems that arise when embedding a high resolution model into the surrounding terrain model. Some inspiration was drawn from existing approaches, which have been however enhanced or generalised. Unlike Latham and Burns' approach, the distant low resolution model remains unchanged and is not influenced by local changes (thus recalling the idea of the "elastic band"). While Warriner and Mandlburger's approach permits a smooth transition of the height profile between two datasets using a chosen weight function, the work presented in this article differs because the overlapping zone is not tied to a buffer of constant width, but it can be of any shape and can potentially have multiple "holes" in it.

In the framework of digital terrain modelling, today's state-ofthe-art DTMs are still 2.5D, and it is present subject of research as how to overcome this limitation in order to include elements such as, for example, overhangs, bridges or tunnels, into a single DTM. Because no strict requirements are given for data inside the inner border, the high resolution object may well be represented by means of a topologically different model: whether another 2.5D DTM, like a nested TIN, or a 2.8D model (Gröger and Plümer, 2005), or a 3D model, this is not explicitly against the initial assumptions; this possibility depends, however, on the capability of the database implementation to handle heterogeneous spatial features. Furthermore, the possibility to leave the high resolution zone unchanged and to insert it into the DTM yields some analogies with the idea of 3D enclaves presented by Otepka et al. (2006).

The experimental tests have shown that the developed methodology works well in the framework of digital terrain modelling, but it can easily be extended to other applications as long as the integration tasks for the datasets are compatible with the prerequisites. In the framework of architectural and archaeological cultural heritage, the possibility of local updates is quite significant, because it is crucial to have a consistent representation and coherent visualisation with regards to the large amounts of available heterogeneous data.

Regarding the simplification of the transition surface, several strategies were investigated in the experimental tests, due to the multiple interpretations that are possible for the simplification parameter $\varepsilon_{\max }$ and according to the availability of information for the surrounding low resolution model. The first method $\left(\varepsilon_{\max }=\sigma_{H}\right)$ is preferred whenever accuracy information is available. This is, however, not always the case. For example, the low resolution model might be a derivative product where accuracy information is not available or has been lost for some reason in the data processing pipeline (Goodchild, 2008). It is not uncommon that existing point cloud processing software offers tools (e.g. for mesh editing, point decimation, etc.) that sometimes operate as black boxes and where little control is left to the user. For such scenarios, the second method seeks a characterising parameter of the surrounding low resolution model using only geometric information. In case even this method fails, a heuristic approach is instead required.

\subsection{Further improvements}

Although the results show that the methodology delivers good results, several initial assumptions are required and some important decisions must be made by the user. Moreover, some existing limitations need to be overcome if the number of assumptions 
regarding the datasets to be integrated is to be reduced and the applicability of this work extended to more general datasets.

Currently, the adoption of a full 3D approach is hindered, for one, by the height difference between the two input meshes because it is calculated on the $z$-axis, which restricts the datasets to be only $2.5 \mathrm{D}$ in the overlapping zone C. This approach is actually well-accepted in the framework of DTM, but yields some limitations when it has to be applied to geometrically more complex surfaces. Secondarily, the normalised distance parameter $d_{n}$ is obtained from buffers created on the $x y$-plane. This is a reasonable approximation as long as the planar annular domain of the overlapping zone does not differ too much from the overlying polyhedral surface. An improvement should consider calculating geodesic shortest paths on the actual polyhedral surface. In Surazhsky et al. (2005), for example, several practical methods to compute both exact and approximate distances on a triangle mesh are presented. The geodesic paths can even cut across the faces in the mesh, unlike the traditional graphbased Dijkstra algorithm for shortest paths. However, in the specific case of the transition surface, applying one of these improved distance functions may lead to a circular problem, because geodesic distances should be calculated on a surface that has not yet been calculated, since the distance parameter for the height interpolation model is first required. A possible solution to this problem could reside in an iterative (and hopefully convergent) procedure.

When it comes to the simplification algorithm, the current implementation lacks direct control over the vertex removal. A further check for this operation needs to be implemented, in order to stop the simplification at an intermediate level before all possible vertices are simplified. Besides the existing geometrical constraints, an additional condition should stop the removal operation before it causes point density to drop beyond a certain threshold. Additionally, the threshold should vary according to the distance from the inner to outer border where the respective point density values must be respected. It must be noted that the term "point density" has been loosely used, i.e. without a specific definition. However, while the concept of point density is straightforward when referred to the $x y$-plane, this is not the case when considering the point density on the mesh surface, being itself an approximation of the (unknown) real surface. In this case, a possible solution to the computation of point density could consider the mutual distances among connected points on the mesh.

One major planned improvement of the methodology resides in a general overhaul of the current deterministic approach with a stochastic one that accounts for spatial data uncertainty. The current methodology requires that the input datasets be aligned, and then assumes that all resulting errors from this operation are negligible. In reality, this simplification does not hold true and systematic errors should be indeed considered and incorporated into the model. Along these lines, a stochastic approach should help to distinguish between systematic and random errors.

More generally, uncertainty is part of a wider group of quality parameters that are commonly referred to as Spatial Data Quality (SDQ). To this extent, at least two further problems need to be addressed: (a) how to store variation of spatial data quality in a database and (b) how to calculate variation of spatial data quality for different GIS primitives such as points, lines and polygons. For the former problem, several solutions exist. One option is that SDQ information can be stored separately from the spatial database through links, it is however difficult to update, and often contains only aggregated quality values for the entire spatial database, thus ignoring de facto spatial variation. Another option is to integrate SDQ into the spatial database, thus letting the user to represent and query more easily spatially varying quality on a per feature basis.

However, variation within an object, sometimes defined as sub-feature variation, cannot be represented in either of these approaches. A third, hybrid approach has been recently proposed by Sadiq et al. (2009), which tries to overcome this drawback, but the overall problem is still unresolved. Questions remain as to how to calculate variation of SDQ for different GIS primitives. Provided that standard deviation values are given for the vertices of a polyline (or a polygon), what happens to the points inside these objects? Picking for example a line segment, it can be easily shown by error propagation that, if the coordinates of the endpoints are spatially uncorrelated and hold the same standard deviation, the standard deviation decreases along the segment and reaches its minimum at the midpoint. One would expect the value to increase as long as it moves further from the known measured endpoints, and eventually to reach its maximum at the midpoint. This apparent paradox is well known is the GIS community and has been subject of research by several authors. In practice, the segment line is fictional and deviations of the truth from the straight line will tend to grow away from the endpoints, more than compensating for this effect (Goodchild, 2008). A solution may come from the adoption of geostatistical interpolation algorithms like kriging, which permits users to estimate prediction errors and take spatial correlation into account. However, with regards to the transition surface, this approach would not necessarily reduce user intervention: obtaining a correct empirical variogram requires, for example, that parameters like the lag size, the variogram fitting model or the definition of the searching neighbourhood are set. These and other open issues are discussed in Karel et al. (2006), but it suffices here to say that the variogram is calculated on the basis of distances obtained on the $x y$-plane, and its parameterisation on top of a triangular mesh embedded in 3D space is theoretically possible, but not immediately straightforward. A final improvement to the methodology proposed in this article is to overcome the initial requirement that the collar borders be given as input parameters.

\subsection{Outlook}

Assuming that most of these unresolved issues will be eventually resolved, three topics are suggested for future avenues of investigation. In the framework of digital terrain modelling, it could be worthwhile to study how to integrate multiple zonally fragmented datasets. A border point may be shared, for example, by three countries having each a DTM with different characteristics. It would then be useful to extend the current dual approach (high resolution vs. low resolution) to a more complex, simultaneous combination of multiple datasets. It has been previously hinted at the analogies between signal processing and the characterisation of a DTM, so it could be worthwhile to create a variable low-pass filter for the transition surface using techniques from this field, although spectral analysis may not have found its way to mainstream practice in the framework of digital terrain modelling.

Finally, as data integration problems in the framework of cultural heritage bear many common traits with those of the DTMs, it could be extremely useful to integrate heterogeneous 3D models with a fully 3D approach that preserves topology, geometric continuity, and guarantee a gradual transition between the surfaces of the different models.

\section{Acknowledgements}

The research work presented in this article has been carried out in great part thanks to scholarships granted by Fondazione Cassa di Risparmio di Padova e Rovigo and by DAAD (Deutscher Akademischer Austausch Dienst).

The authors would like to thank Christian Manthe (Technical University of Berlin), who helped to acquire the underground entrance in Berlin, the members of the Laboratorio di Rilevamento 
e Geomatica (University of Padua) who have acquired the datasets in Nora, and the article reviewers for their constructive remarks and helpful suggestions.

\section{References}

Agugiaro, G., 2009. Advanced methodologies of acquisition, integration, analysis, management, visualisation and distribution of data in the framework of archaeological and architectonical heritage. Ph.D. thesis, Università di Padova and Technische Universität Berlin.

Agugiaro, G., Kolbe, T.H., 2009. Definition of a transition surface with the purpose of integration between a laser scanner 3D model and a low resolution DTM. International Archives of Photogrammetry, Remote Sensing and Spatial Information Sciences 38 (Part 5/W1) (on CD-ROM).

Agugiaro, G., Salemi, G., Achilli, V., Cervato, C., Cuppari, F., 2008. Il rilievo lase scanner con texturizzazione HDR dei portali della chiesa di Mattia a Budapest. Recupero e conservazione, deLettera Editore 83, 40-45.

Bejor, G., 1992. Nora I, L'abitato romano: distribuzione, cronologie, sviluppi. Soprintendenza Archeologica per le province di Cagliari e Oristano, Quaderni 9, 125-132.

Cohen, J., Varshney, A., Manocha, D., Turk, G., Weber, H., Agarwal, P.K., Brooks, Jr F.P., Wright, W.V., 1996. Simplification envelopes. in: Proc. of Siggraph, New Orleans, LA, pp. 119-128.

Doytsher, Y., Filin, S., Ezra, E., 2001. Transformation of datasets in a linear-based map conflation framework. Surveying and Land Information Systems 61 (3), 165-175.

Fabris, M., Boatto, G., Achilli, V., 2011. 3D laser scanning surveys in the modelling of cultural heritage. In: Meola, C. (Ed.), Recent Advances in Non-Destructive Inspection. Nova Science Publishers, pp. 1-32.

Felus, Y.A., Csathó, B., 2000. Multi-source DEM evaluation and integration at the Antarctica transantarctic mountains project. International Archives of Photogrammetry and Remote Sensing 33 (Part B1), 117-123.

Förstner, W., Plümer, L., 1997. Semantic Modeling for the Acquisition of Topographic Information from Images and Maps: SMATI 97. Birkhäuser, Basel.

Gielsdorf, F., Gründig, L., 1997. Nachbarschaftstreue anpassung auf der basis des membranmodells. Zeitschrift für Vermessungswesen 1997 (5), 208-218.

Goodchild, M.F., 2008. Imprecision and spatial uncertainty. In: Shekhar, S., Xiong, H. (Eds.), Encyclopedia of GIS. Springer, pp. 480-483.

Gröger, G., Plümer, L., 2005. How to get 3-D for the price of 2-D. Topology and consistency of 3-D Urban GIS. Geoinformatica 9 (2), 139-158.

Gründig, L., Gielsdorf, F., Aschoff, B., 2007. Merging different data sets based on matching and adjustment techniques. Strategic Integration of Surveying Services, FIG Working Week, Hong Kong (on CD-ROM).

Guidi, G., Frischer, B., Lucenti, I., 2007. Rome reborn - virtualizing the ancient imperial Rome. in: Remondino, F., El-Hakim, S., Gonzo, L. (Eds.), 3D-Arch'07,
International Archives of Photogrammetry, Remote Sensing and Spatial Information Sciences 36 (Part 5/W47) (on CD-ROM).

Haunert, J.-H., 2005. Link based conflation of geographic datasets. in: Proc. 8th ICA Workshop on Generalisation and Multiple Representation, A Coruña, Spain (on CD-ROM).

Kampshoff, S., 2005. Mathematical models for geometrical integration. in: Gröger, G., Kolbe, T.H. (Eds.), First International Workshop on Next Generation 3D City Models. Bonn, Germany, EuroSDR Publication No. 49.

Karel, W., Pfeifer, N., Briese, C., 2006. DTM quality assessment. in: ISPRS Technical Commission II Symposium 2006, International Archives of Photogrammetry. Remote Sensing and Spatial Information Sciences 36 (Part 2), pp. 7-12.

Katzil, Y., Doytsher, Y., 2005. Spatial rubber sheeting of DTMs. in: Proc. 6th Geomatics Week Conference, February 2005, Barcelona.

Koch, A., Heipke, C., 2006. Semantically correct 2.5D GIS data - the integration of a DTM and topographic vector data. ISPRS Journal of Photogrammetry and Remote Sensing 61 (1), 23-32.

Kolbe, T.H., 2008. Representing and exchanging 3D city models with cityGML. in: Lee, Zlatanova (Eds.), 3rd International Workshop on 3D Geoinformation, Seoul, Korea. Lecture Notes in Geoinformation and Cartography, Springer, pp. 15-31.

Latham, R., Burns, D., 2006. Dynamic terrain modification using a correction algorithm. in: Proc. Image 2006 Conference, Scottsdale, Arizona, USA.

Laurini, R., 1998. Spatial multi-database topological continuity and indexing: a step towards seamless GIS data interoperability. International Journal of Geographical Information Science 12 (4), 373-402.

Luebke, D., Reddy, M., Cohen, J., Varshney, A., Watson, B., Huebner, R., 2002. Level of Detail for 3D Graphics. Morgan Kaufmann Publishing.

Otepka, J., Briese, C., Nothegger, C., 2006. First steps to a topographic information system of the next generation. International Archives of Photogrammetry. Remote Sensing and Spatial Information Sciences 36 (Part 4) (on CD-ROM)

Sadiq, M.G.S., Ghouse, M., Duckham, M., 2009. Integrated storage and querying of spatially varying data quality information in a relational spatial database. Transactions in GIS 13 (1), 25-42.

Shekhar, S., Xiong, H., 2008. Encyclopedia of GIS. Springer.

Shewchuk, J.R., 1996. Triangle: engineering a 2D quality mesh generator and delaunay triangulator. in: Ming, Lin, Dinesh (Eds.), Applied Computational Geometry: Towards Geometric Engineering, vol. 1148 of Lecture Notes in Computer Science, Springer, Berlin, pp. 203-222.

Stadler, A., Kolbe, T.H., 2007. Spatio-semantic coherence in the integration of 3D city models. International Archives of Photogrammetry, Remote Sensing and Spatial Information Sciences 36 (Part 2/C43), on CD-ROM.

Surazhsky, V., Surazhsky, T., Kirsanov, D., Gortler, S.J., Hoppe, H., 2005. Fast exact and approximate geodesics on meshes. ACM Transactions on Graphics 24 (3), 553-560.

Warriner, T., Mandlburger, G., 2005. Generating a new high resolution DTM product from various data sources. in: Fritsch, D. (Ed.), Proc. 50th Photogrammetric week, Wichmann Verlag, Heidelberg, pp. 197-206. 